\title{
Effect of rs67085638 in long non-coding RNA (CCAT1) on colon cancer chemoresistance to paclitaxel through modulating the microRNA-24-3p and FSCN1 signaling
}

Caihong Zhang

Xing Yuan Hospital of Yulin

Yonglin Wang ( $\square$ heartscience@yeah.net)

Yangling Dempnstration Zone Hospital https://orcid.org/0000-0002-4419-0593

Primary research

Keywords: Colon cancer, CCAT1, SNP, miR-24-3p, FSCN1, Paclitaxel

Posted Date: May 22nd, 2020

DOI: https://doi.org/10.21203/rs.3.rs-24077/v1

License: (a) (1) This work is licensed under a Creative Commons Attribution 4.0 International License.

Read Full License 


\section{Abstract}

\section{Background}

It has been reported that rs67085638 in IncRNA-CCAT1 was associated with the risk of tumorigenesis. Also, CCAT1 could affect chemoresistance of cancer cells to PTX via regulating miR-24-3p and FSCN1 expression. In this study, we aimed to investigate the effect of rs67085638 on the expression of CCAT1/miR-24-3p/FSCN1 and the response of colon cancer to the treatment of PTX.

Method

48 colon cancer patients were recruited and grouped by their genotypes of rs 67085638 polymorphism as a CC group ( $\mathrm{N}=28)$ and a CT group $(\mathrm{N}=20)$. Colon cancer cells were collected from the patients and cancer cell xenografts were transplanted into mice. PCR analysis, IHC assay and Western blot were performed to observe the expression of IncRNA-CCAT1, miR-24-3p and FSCN1 in vivo and in vitro, and the relationships among the expression of IncRNA-CCAT1, miR-24-3p and FSCN1 were validated by computational analysis and luciferase assay. TUNEL assay and flow cytometry were conducted to observe tumor cell apoptosis and survival.

Result

LncRNA-CCAT1 and FSCN1 mRNA/protein were over-expressed while miR-24-3p was down-regulated in the CT-genotyped patients and cells compared with those in the CC-genotyped patients and cells. The survival of colon cancer cells was decreased while the apoptosis of colon cancer cells was increased by PTX treatment in a dose-dependent manner. However, the survival rate of CT-genotyped cells was higher while the apoptosis rate of CT-genotyped cells was lower than that of the CC-genotyped cells, and the difference was partly eliminated by the knockdown of IncRNA-CCAT1. MiR-24-3p was validated to target IncRNA-CCAT1 and FSCN1 mRNA, and the over-expression of CCAT1 could reduce the expression of miR24-3p while elevating the expression of FSCN1. The growth of CT-genotyped tumors in mice was more suppressed in compared with the growth of CC-genotyped tumors, while the knockdown of IncRNACCAT1 partly reversed the effect of the CT genotype. Furthermore, compared with the rs67085638-CC mice, the IncRNA-CCAT1 and FSCN1 mRNA/protein levels in the rs67085638-CT+NC shRNA mice were increased while their miR-24-3p level was decreased, and the knockdown of IncRNA-CCAT1 partly reversed the dysregulation of these genes.

Conclusion

The findings of this study demonstrated that the presence of the minor allele of rs 67085638 increased the expression of CCAT1 and accordingly enhanced the resistance to PTX. Downregulation of CCAT1 partially, but significantly, re-stored the sensitivity to PTX of colon cancer cells.

\section{Background}


Colon cancer is a type of common malignant tumors in the digestion system that occurs mainly at the junction of the sigmoid colon and rectum, with the greatest incidence in people of 40-to-50 years old [1]. Colon cancer attributes to $1 / 3$ of all malignant tumor cases worldwide. Colon cancer is mainly separated into undifferentiated carcinoma, mucinous adenocarcinoma, as well as adenocarcinoma. The general form of colon cancer is ulcers or polypoid [2]. Individuals with colon polyps or persistent colitis, as well as obese males are predominantly vulnerable to colon cancer [3]. While non-specific cytotoxicity reduces the therapeutic index of colon cancer, causing small differences in treatment efficacy, the resistance to 5-FU treatment is commonly seen to result in a poor prognosis [4].

With its broad spectrum anti-tumor functions as well as its capacity to prevent angiogenic features of endothelial cells, paclitaxel seems like a strong prospective agent for chemotherapy $[5,6]$. All current results showed that the mix of a low-dose therapy of paclitaxel with the knockdown of hypoxia-inducible factor-1a may induce a potent inhibitory effect against colon cancer cells [7].

Long non-coding RNAs (IncRNAs) are a type of non-coding RNAs containing more than 200 nucleotides. LncRNAs were reported to play essential roles as epigenetic mediators of gene expression $[8,9]$. More evidence indicated that the dysregulated IncRNA expression is associated with the pathogenesis of various disorders such as cancers [10]. The polymorphisms in IncRNAs may show numerous impacts on the expression as well as functions of IncRNAs, therefore regulating the cancer susceptibility of individuals [11]. Colorectal cancer associated transcript 1 (CCAT1) is an IncRNA of about $11 \mathrm{~kb}$, and the over-expression of CCAT1 appears in both the tumorigenesis and progression of CRC [12]. Xiang et al. actually showed that CCAT1 played a key role in the transcriptional regulation of MYC as well as in promoting chromatin looping. The knock-down of CCAT1 decreases the interaction between the promoter of MYC with its enhancer [13]. Cell growth as well as MYC transcription is strongly correlated with the expression of CCAT1 mRNA in multiple types of tumors [14].

MiR-24-3p is an miRNA whose role has been ignored in cardiac I/RI. In the last few years, miR-24-3p has been extensively studied as a tumor suppressor in many types of tumors, including nasopharyngeal carcinoma, liver cancer, as well as lung cancer, indicating that miR-24-3p contributes in the process of tumor growth suppression as well as enhancing the apoptosis of tumor cells $[15,16]$. It was actually presented that the expression of miR-24-3p was negatively moderated via CCAT1, while the depletion of miR-24-3p eliminated the suppressive impact of knockdown of CCAT1 on PTX resistance, suggesting that CCAT1 managed PTX resistance through sponging the expression of miR-24-3p [17]. Li et al have shown that miR-24 could reduce the migration, proliferation, invasion as well as growth of tumor cells through targeting the FSCN1 expression in nasopharyngeal cancer [17].

As an actin binding protein with a molecular weight of $55 \mathrm{kDa}$, FSCN1 works as a significant factor in keeping the stability of filamentous actins. FSCN1 arranges F-actins in cells to create tightly packed bundles of F-actins to assist the formation of certain primary structures based on actin: Cortical cell projections that moderate cell-cell communications as well as cell migration, as well as cytoplasmic microfilaments that aid the formation of cell structures and intracellular movement $[18,19]$. FSCN1 has 
recently become a focus of research as a novel biomarker for many types of cancers. FSCN1 showed reduced expression in normal epithelial cells, but its expression is increased in esophageal cancer, lung cancer, breast cancer, as well as prostate cancer $[18,20]$.

It has been reported that a single nucleotide polymorphism (rs67085638) in IncRNA-CCAT1 was associated with the risk of tumorigenesis [21]. It was additionally presented that the rs67085638 C > T SNP in IncRNA CCAT1 was linked to an enhanced risk of colon cancer [21]. Another study showed that CCAT1 may affect the chemoresistance of ovary cancer cells to PTX via regulating miR-24-3p and FSCN1 [17]. In this study, we investigated the effect of rs 67085638 on the expression of CCAT1/miR-24$3 p / F S C N 1$ and the response of colon cancer to the treatment of PTX.

\section{Materials And Methods}

\section{Patient recruitment}

In this study, we recruited 48 colon cancer patients seeking treatment at our hospital. The tumor tissues of these patients were collected for genotyping of the rs 67085638 polymorphism (see below). Based on the results of genotyping of the rs67085638 polymorphism, the patients were grouped into a CC group $(\mathrm{N}=28)$ and a CT group $(\mathrm{N}=20)$. The demographic and clinicopathological characteristics of each patient were also collected and compared between the two patient groups. The Human Research Ethics Committees of Yangling Demonstration Zone Hospital has approved this research and all methods were performed in accordance with the last vision of the Declaration of Helsinki.

\section{Animal model}

Cancerous colon cells were collected from the colon cancer patients genotyped as CC or CT. Then, three cell groups were set up, i.e., an rs67085638-CC group (cancerous colon cells genotyped as CC), an rs67085638-CT+NC shRNA group (cancerous colon cells genotyped as CT and then treated with NC shRNA), and an rs67085638-CT+IncRNA-CCAT1 group (cancerous colon cells genotyped as CT and then treated with IncRNA-CCAT1). The mice were also divided into three groups corresponding to the three groups of cells, i.e., 1. rs67085638-CC mice; 2. rs67085638-CT+NC shRNA mice; and 3. rs67085638CT+shRNA-CCAT1 mice, to generate a mouse model of colon cancer, which was then treated with PTX at a dose of $12 \mathrm{mg} / \mathrm{kg}$ injected via the intravenous injection through the tail every three days for a total of 21 days to study the expression of target genes. In brief, 4-week old BALB/c male nude mice with a body weight of $19 \pm 1 \mathrm{~g}$ were purchased from the animal facility of our hospital. This research study was approved by the Ethics Committee of our hospital. The mice were given unlimited access to a standard pellet diet as well as unlimited drinking water. The mice were placed in an airy room with a regulated temperature of $25 \pm 2{ }^{\circ} \mathrm{C}$ and a $12 \mathrm{~h} / 12 \mathrm{~h}$ dark/light cycle. After 7 days of adaptation, the mice were arbitrarily divided in 2 groups ( $\mathrm{n}=15$ in each group). Then, the cells described above were administered via subcutaneous injection into the rear side of each mouse. After 21 days of treatment, the mice were killed through deep anaesthesia and the tumor in each mouse was removed to measure its weight as well as volume by making use of the formula: $V=(W \times W \times L) / 2$, where $V$ was the tumor volume, $W$ was 
the tumor width, while $L$ was the tumor length. All animal experiments were performed in line with the Guide for the Care and Use of Laboratory Animal by International Committees and were approved by the Animal Ethics Committee of Yangling Demonstration Zone Hospital.

\section{Cell culture and transfection}

Primary colon cancer cells were isolated from colon cancer patients and maintained in a modified Dulbecco's Eagle medium (DMEM) supplemented with $10 \%$ of fetal bovine serum and antibiotics of trypsin and streptomycin (Gibco, Thermo Fisher Scientific, Waltham, MA). Then, the cells were divided into 3 groups, i.e., 1. rs67085638-CC group; 2. rs67085638-CT+ NC shRNA; and 3. rs67085638-CT+ shRNA-CCAT1. In the next step, the cells in groups rs67085638-CT+ NC shRNA and rs67085638-CT+ shRNA-CCAT1 were transfected with corresponding shRNAs using Lipofectamine 2000 (Invitrogen, Carlsbad, CA) according to the instructions provided by the manufacturer. At $48 \mathrm{~h}$ after transfection, the cells were collected to assay the expression of target genes. In addition, some of the cells were treated with different concentrations of PTX, and the survival status of the cells was measured using an MTT assay. In brief, primary colon cancer cells were treated with different concentrations of PTX for two days. Then, an MTT assay kit bought from Aladdin (Shanghai, China) was used according to the instructions provided by the manufacturer. Ultimately, the absorbance in each well was read at a wavelength of 490 $\mathrm{nm}$ by utilizing a microplate reader (Bio-Rad, Hercules, CA) to plot the survival curve and calculate the survival of cells at different time points, thus calculating the IC50 of PTX.

\section{RNA isolation and real-time PCR}

Collected tumor tissues and cell samples were treated with Trizol (Invitrogen, Carlsbad, CA) according to the instructions provided by the manufacturer to extract total RNA of each sample. Then, a QuantScript reverse transcription assay kit (Tiangen Biotech, Shanghai, China) was used for 60 minutes of reverse transcription at $37^{\circ} \mathrm{C}$ to obtain $\mathrm{CDNA}$, which was subjected to real time PCR by using a qPCR assay kit (Tiangen Biotech, Shanghai, China) according to the instructions provided by the manufacturer. The real time PCR reactions were carried out in a Step OnePlus Real-Time PCR machine. Finally, the relative expression of IncRNA-CCAT1, miR-24-3p, and FSCN1 mRNA in each sample was calculated by using the $2^{-\Delta \Delta \mathrm{Ct}}$ approach.

\section{Luciferase assay}

The Target Scan software was used to predict the binding sites of miR-24-3p on FSCN1 mRNA and IncRNA-CCAT1. Then, wild type IncRNA-CCAT1 sequence and 3' UTR of FSCN1 mRNA containing the miR24-3p binding sites were respectively cloned into pcDNA3.1 vectors (Promega, Madison, WI) to create wild type IncRNA-CCAT1 and FSCN1 mRNA vectors. At the same time, a Quick Change mutagenesis kit (Stratagene, San Diego, CA) was used to create site directed mutations in the miR-24 binding sites of IncRNA-CCAT1 and FSCN1 mRNA, and the mutated sequences were also respectively cloned into pcDNA3.1 vectors to create mutant type IncRNA-CCAT1 and FSCN1 mRNA vectors. Finally, CACO-2 cells were co-transfected with IncRNA-CCAT1 and miR-24-3p mimics or FSCN1 mRNA and miR-24 -3p mimics, 
and a dual-luciferase reporter gene assay kit (Promega, Madison, $\mathrm{Wl}$ ) was used $24 \mathrm{~h}$ later according to the instructions provided by the manufacturer to determine the luciferase activity of transfected cells.

\section{Survival rate}

The rates of apoptosis and survival were determined with FCM analysis by making use of an Annexin VAPC/PI Apoptosis Assay kit (KeyGen Biotech, Nanjing, China) according to the instructions provided by the manufacturer. The reading of results was done on a FACS Canto II flow cytometer (BD Biosciences, San Jose, CA) utilizing the FlowJo X.10.0.7-1 software.

\section{Western blot analysis}

The expression level of FSCN1 in each sample was determined through Western blotting. In brief, the samples were lysed in a radioimmunoprecipitation buffer to extract total protein, which was then resolved on $10 \%$ SDS-PAGE. In the next step, the resolved proteins were electrophoretically blotted onto polyvinylidene fluoride (PVDF) membranes, which were then blocked with $5 \%$ skim milk at ambient temperature for $1 \mathrm{~h}$. Then, the membranes were incubated along with anti-FSCN1 (1:1000, Abcam, Cambridge, MA) primary antibodies and HRP-labeled secondary antibodies under conditions suggested by the antibody manufacturer. Finally, the membranes were developed in an enhanced chemiluminescence $(E C L)$ reagent (Thermo Fisher Scientific, Waltham, MA) and the densitometry analysis of protein bands was done using ImageJ software to determine the relative protein expression of FSCN1 in each sample.

\section{IHC assay}

The collected tissue samples were fixed by using $10 \%$ neutral formalin (Thermo Fisher Scientific, Waltham, MA) according to the instructions provided by the manufacturer. After embedding in paraffin and partitioning into $5 \mu \mathrm{m}$ slices, the tissue sections were dewaxed, rehydrated and subject to antigen retrieval. Then, the slides were blocked by using bovine albumin and treated with anti-FSCN1 primary antibodies and biotin labeled secondary antibodies under conditions suggested by the antibody manufacturer. Finally, the slides were observed under a fluorescence microscope to determine the positive expression rate of FSCN1 in each sample.

\section{TUNEL assay}

Cell apoptosis was analyzed by using a terminal deoxynucleotidyl transferase-mediated X-dUTP nick end labeling (TUNEL) assay kit (Roche, Basel, Switzerland) according to the instructions provided by the manufacturer.

\section{Statistical analysis}

All statistical analyses were done by utilizing GraphPad Prism 7.0 software (GraphPad, La Jolla, CA). All results were presented as the mean \pm standard errors. Comparisons of between-group differences were 
done by making use of Student's $t$ tests. $P<0.05$ was taken into consideration to show a statistically significant difference.

\section{Results}

\section{Colon cancer patient recruitment}

In this study, we recruited 48 colon cancer patients and grouped them by their genotypes of the rs67085638 polymorphism as a CC group $(\mathrm{N}=28)$ and a CT group $(\mathrm{N}=20)$. We measured the demographic and clinicopathological characteristics of each patient, and collected and demonstrated the results in Table 1. Accordingly, we found no evident differences of these characteristics among these two patient groups.

\section{Gene expression in colon cancer patients}

Expression of target genes of this study, including IncRNA-CCAT1, miR-24-3p, and FSCN1 mRNA, was observed in the cancerous tissue samples. Accordingly, compared with the CC-genotyped patients, the CTgenotyped patients showed markedly up-regulated expression of IncRNA-CCAT1 (Fig.1A) and FSCN1 mRNA (Fig.1C) along with evidently down-regulated expression of miR-24-3p (Fig.1B). Moreover, we observed an evident increase of FSCN1 protein (Fig.2) expression in the CT group compared with that in the CC group.

\section{Gene expression in colon cancer cells of different genotypes}

Cancerous colon cells collected from the colon cancer patients genotyped as CC or CT were collected. Three cell groups were set up. Compared with the rs67085638-CC group, we observed an evident increase of IncRNA-CCAT1 (Fig.3A) and FSCN1 mRNA (Fig.3C) expression, as well as a significant decrease of miR-24-3p (Fig.3B) expression, in the rs67085638-CT+shRNA-CCAT1 group. And the knockdown of IncRNA-CCAT1 by CCAT1 shRNA resulted in suppressed IncRNA-CCAT1 (Fig.3A) and FSCN1 mRNA (Fig.3C) expression along with increased miR-24-3p (Fig.3B) expression in CT-genotyped cells. Also, Western blot analysis validated the changes of FSCN1 protein (Fig.3D) expression in different groups of colon cancer cells.

\section{Effects of PTX treatment on the survival and apoptosis of colon cancer cells}

The colon cancer cells of different genotypes were subjected to PTX treatment at concentrations of $1 \mathrm{nM}$, $1.4 \mathrm{nM}, 1.8 \mathrm{nM}, 2.2 \mathrm{nM}, 2.6 \mathrm{nM}, 3.0 \mathrm{nM}$ and $3.4 \mathrm{nM}$. The survival and apoptosis rates of each cell group were measured by flow cytometry, respectively. As shown in Fig.4A, the survival of each cell group was decreased by the PTX treatment in a dose-dependent manner. However, the survival rate of CT-genotyped cells was higher than that of the CC-genotyped cells, while the knockdown of IncRNA-CCAT1 in CTgenotyped cells suppressed their survival. On the contrary, as shown in Fig.4B, the apoptosis rate of CTgenotyped cells was lower than that of the CC-genotyped cells. Additionally, the survival rate of the rs67085638-CT+NC shRNA group was higher than that of the rs67085638-CT+shRNA-CCAT1 group. 


\section{Establishment of a CCAT1/miR-24-3p/FSCN1 signaling pathway}

Online bioinformatic tools were utilized to compare the sequences of IncRNA-CCAT1, miR-24-3p, and FSCN1 mRNA. Accordingly, as shown in Fig.5A, we detected a putative binding site of miR-24-3p on IncRNA-CCAT1, and the subsequent luciferase assay showed markedly reduced luciferase activity of wildtype IncRNA-CCAT1 in CACO-2 cells transfected with miR-24-3p mimics. Additionally, as shown in Fig.5B, a binding site was also detected in the 3'UTR of FSCN1 mRNA, and the luciferase activity was significantly reduced in CACO-2 cells co-transfected with miR-24-3p and wild type FSCN1 3'UTR, indicating that miR-24-3p could target the expression of FSCN1 mRNA in CACO-2 cells.

CACO-2 cells were subsequently transfected with empty pcDNA3 vectors (pcDNA3 group) or pcDNA3 vectors carrying CCAT1 (pcDNA3-CCAT1 group). As shown in Fig.5C, the expression of miR-24-3p was reduced (Fig.5C) while the expression of FSCN1 was elevated (Fig.5D and 5E) in the pcDNA3-CCAT1 group compared with that in the pcDNA3 group.

\section{Xenograft study of colon tumors}

The colon tumor cells were transplanted to mice to establish different groups of mice: rs67085638-CC mice, rs67085638-CT+NC shRNA mice and rs67085638-CT+shRNA-CCAT1 mice. The xenograft in each group of mice was studied, and the tumor volume and tumor cell apoptosis rate were measured. Accordingly, the mouse tumor volume was the highest in the rs67085638-CT+NC shRNA mice and the lowest in the rs67085638-CC mice (Fig.6A). Also, the tumor cell apoptosis rate was the highest in the rs67085638-CC mice and the lowest in the rs67085638-CT+NC shRNA mice (Fig.6B), thus validating that the tumor growth was more suppressed in CT-genotyped tumors, while the knockdown of IncRNA-CCAT1 partly reversed the effect of the CT genotype.

Furthermore, compared with the rs67085638-CC mice, the expression levels of IncRNA-CCAT1 (Fig.7A) and FSCN1 mRNA (Fig.7C)/protein (Fig.7D) were significantly increased in the rs67085638-CT+NC shRNA mice, and the knockdown of IncRNA-CCAT1 partly reversed the dysregulation of IncRNA-CCAT1 and FSCN1 mRNA/protein. On the contrary, as shown in Fig.7B, miR-24-3p was decreased in the rs67085638-CT+NC shRNA and rs67085638-CT+shRNA-CCAT1 mice, with the rs67085638-CT+NC shRNA mice showing the lowest level of miR-24-3p.

\section{Discussion}

In this study, we collected tissue samples form OC patients who were sensitive or blunt to the treatment of PTX. We found that IncRNA-CCAT1 and FSCN1 mRNA/protein were up-regulated, while the level of miR-24-3p was down-regulated in the CT group compared with that in the CC group. Furthermore, we also found that the survival of colon cancer cells was decreased while the apoptosis of colon cancer cells was increased by the PTX treatment in a dose-dependent manner. However, the survival rate of CT-genotyped cells was higher while the apoptosis rate of CT-genotyped cells was lower than that of the CC-genotyped cells, and the effects of the CC-genotype were partly blocked by the knockdown of IncRNA-CCAT1. It was 
revealed that the distribution of 3 SNPs, rs67085638, rs7013433, as well as rs77628730, in the CCAT1 gene was associated with the risk of CRC. However, even in the presence of such association, only the polymorphism of rs 67085638 was discovered to be associated with an elevated CRC risk after adjusting for the age, education level, sex, BMI, as well as the ratio of red meat product and root vegetables in the diet. In fact, the polymorphism of rs 67085638 was discovered to be associated with an elevated colon cancer risk but not an elevated rectal cancer risk [21]. Another research showed in line results by revealing that the polymorphism of rs67085638 in the 3'UTR of CCAT1 was linked to elevated colon cancer and rectal cancer risks [21]. One team of scientists presented that as compared to healthy cells, CCAT1 was confirmed to become overexpressed in colon tumor cells to enhance the invasion as well as proliferation of colon tumor cells. In fact, CCTA1 is actually associated with the metastasis of lymph nodes as well as patient prognosis [22]. Sun et al. showed that CCAT1 was a prospective biomarker of colonic tumors, which showed that CCAT1 may be made use of to estimate the prognosis of colon cancer [23]. It was likewise shown that the IC50 value of PTX was reduced in PC3-TXR cells than in DU145-TXR cells, suggesting that DU145 cells displayed higher resistance to PTX [24]. Significantly, the study on PTX resistance showed that the presence of CCAT1 lowered the IC50 value of PTX as well as cell viability yet boosted the apoptosis of both DU145-TXR and PC3-TXR cells [25]. In this study, the CCAT1/miR-24$3 p / F S C N 1$ signaling pathway was established by validating the targets of miR-24-3p. The overexpression of CCAT1 could reduce the expression of miR-24-3p while elevating the expression of FSCN1 mRNA and protein. In addition, we also found that, compared with the rs67085638-CC group, the rs67085638-CT + shRNA-CCAT1 group showed an evident increase of IncRNA-CCAT1 and FSCN1 mRNA/protein expression and a significant decrease of miR-24-3p expression, and the effects of the CCgenotype were partly blocked by the knockdown of IncRNA-CCAT1.

Previous research revealed that the prospective downstream targets of CCAT1 included miR-24-3p, which may bind to CCAT1 in RNA pull-down, RIP as well as luciferase assays [17].

The bioinformatics evaluations illustrated the prospective binding site of miR-24-3p in FSCN1. Subsequently, the presence of miR-24-3p caused a reduction of luciferase activity in both DU145-TXR and PC3-TXR cells. In addition, miR-24-3p over-expression led to even more FSCN1 expression in the cells [17]. Gao et al. [18] displayed that miR-24-3p was actually downregulated in CC cells, but the miR-24-3p overexpression suppressed the proliferation of CC cells. Mishra et al displayed that miR-24 acts as a p53independent tumor suppressor by suppressing the expression of dihydrofolate reductase in CC cells [26]. Moreover, Fang et al revealed that the blood level of miR-24 was reduced in CC patients [27].

Moreover, it was shown that the expression of miR-24-3p was actually increased with the reduced expression of CCAT1 to eliminate the suppressive impact of CCAT1 on PTX resistance, suggesting that CCAT1 controlled PTX resistance through sponging the expression of miR-24-3p [17].

As an evolutionarily preserved actin-binding protein in the filopodia, Fascin 1 is associated with cell migration using actin-based protrusions located below the cell membrane $[19,28]$. Fascin1 participates in cell migration, adhesion as well as invasion in the bowel cancer as well as melanoma, and is associated 
with poor cancer prognosis [29-32]. Additionally, FSCN1 acts as a miR-24-3p target in miR-24-3pmediated sensitivity to PTX [17]. In this study, the tumor growth in mice was more suppressed in CTgenotyped tumors compared with the CC-genotyped tumors, while the knockdown of IncRNA-CCAT1 partly blocked the effects of the CT genotype.

Furthermore, compared with the rs67085638-CC mice, the levels of IncRNA-CCAT1 and FSCN1 mRNA/protein were increased while the miR-24-3p level was decreased in the rs67085638-CT + NC shRNA mice, and the knockdown of IncRNA-CCAT1 partly reversed the dysregulation of these genes.

\section{Conclusion}

The findings of this study demonstrated that the presence of the minor allele of rs 67085638 increased the expression of CCAT1 and enhanced the chemoresistance to PTX, while the downregulation of CCAT1 partially, but significantly, re-stored the sensitivity of colon cancer cells to PTX.

\section{Abbreviations}

CCAT1

Colorectal cancer associated transcript 1

PTX

paclitaxel

\section{Declarations}

\section{Consent for publication}

Written informed consent was obtained from all patients or their first-degree relatives before the surgery.

\section{Competing interests}

The authors declare that they have no competing interests.

\section{Funding}

No funding was received.

\section{Authors' contributions}

Caihong Zhang planned the study; Yonglin Wang collected the study literature; Caihong Zhang and Yonglin Wang performed the experiment and analyzed the experiment data; Caihong Zhang drafted the manuscript and Yonglin Wang improved the final manuscript.

\section{Acknowledgements}


Not applicable.

\section{Availability of data and material}

The data that support the findings of this study are available from the corresponding author upon reasonable request.

\section{References}

1. Dou C, Sun L, Jin X, Han M, Zhang B, Li T. Long non-coding RNA colon cancer-associated transcript 1 functions as a competing endogenous RNA to regulate cyclin-dependent kinase 1 expression by sponging miR-490-3p in hepatocellular carcinoma progression. Tumour Biol. 2017;39:1010428317697572.

2. He X, Tan X, Wang X, Jin H, Liu L, Ma L, Yu H, Fan Z. C-Myc-activated long noncoding RNA CCAT1 promotes colon cancer cell proliferation and invasion. Tumour Biol. 2014;35:12181-8.

3. Zhang P, Lai ZL, Chen HF, Zhang M, Wang A, Jia T, Sun WQ, Zhu XM, Chen XF, Zhao Z, Zhang J. Curcumin synergizes with 5-fluorouracil by impairing AMPK/ULK1-dependent autophagy, AKT activity and enhancing apoptosis in colon cancer cells with tumor growth inhibition in xenograft mice. J Exp Clin Cancer Res. 2017;36:190.

4. Hector S, Prehn JH. Apoptosis signaling proteins as prognostic biomarkers in colorectal cancer: a review. Biochim Biophys Acta. 2009;1795:117-29.

5. Ng SS, Sparreboom A, Shaked Y, Lee C, Man S, Desai N, Soon-Shiong P, Figg WD, Kerbel RS. Influence of formulation vehicle on metronomic taxane chemotherapy: albumin-bound versus cremophor ELbased paclitaxel. Clin Cancer Res. 2006;12:4331-8.

6. Jiang H, Tao W, Zhang M, Pan S, Kanwar JR, Sun X. Low-dose metronomic paclitaxel chemotherapy suppresses breast tumors and metastases in mice. Cancer Invest. 2010;28:74-84.

7. Zhang M, Chen C, Su F, Huang Z, Li X, Li X. Knockdown of Hypoxia-Inducible Factor 1alpha Improved the Efficacy of Low-Dose Metronomic Chemotherapy of Paclitaxel in Human Colon Cancer Xenografts. Technol Cancer Res Treat. 2017;16:609-19.

8. Derrien T, Johnson R, Bussotti G, Tanzer A, Djebali S, Tilgner H, Guernec G, Martin D, Merkel A, Knowles DG, Lagarde J, Veeravalli L, Ruan X, Ruan Y, Lassmann T, Carninci P, Brown JB, Lipovich L, Gonzalez JM, Thomas M, Davis CA, Shiekhattar R, Gingeras TR, Hubbard TJ, Notredame C, Harrow J, Guigo R. The GENCODE v7 catalog of human long noncoding RNAs: analysis of their gene structure, evolution, and expression. Genome Res. 2012;22:1775-89.

9. Kim JH, Lee BB, Oh YM, Zhu C, Steinmetz LM, Lee Y, Kim WK, Lee SB, Buratowski S, Kim T. Modulation of mRNA and IncRNA expression dynamics by the Set2-Rpd3S pathway. Nat Commun. 2016;7:13534.

10. Castellanos-Rubio A, Fernandez-Jimenez N, Kratchmarov R, Luo X, Bhagat G, Green PH, Schneider R, Kiledjian M, Bilbao JR, Ghosh S. A long noncoding RNA associated with susceptibility to celiac 
disease. Science. 2016;352:91-5.

11. Tao R, Hu S, Wang S, Zhou X, Zhang Q, Wang C, Zhao X, Zhou W, Zhang S, Li C, Zhao H, He Y, Zhu S, $\mathrm{Xu}$ J, Jiang Y, Li L, Gao Y. Association between indel polymorphism in the promoter region of IncRNA GAS5 and the risk of hepatocellular carcinoma. Carcinogenesis. 2015;36:1136-43.

12. Nissan A, Stojadinovic A, Mitrani-Rosenbaum S, Halle D, Grinbaum R, Roistacher M, Bochem A, Dayanc BE, Ritter G, Gomceli I, Bostanci EB, Akoglu M, Chen YT, Old LJ, Gure AO. Colon cancer associated transcript-1: a novel RNA expressed in malignant and pre-malignant human tissues. Int $\mathrm{J}$ Cancer. 2012;130:1598-606.

13. Xiang JF, Yin QF, Chen T, Zhang Y, Zhang XO, Wu Z, Zhang S, Wang HB, Ge J, Lu X, Yang L, Chen LL. Human colorectal cancer-specific CCAT1-L IncRNA regulates long-range chromatin interactions at the MYC locus. Cell Res. 2014;24:513-31.

14. McCleland ML, Mesh K, Lorenzana E, Chopra VS, Segal E, Watanabe C, Haley B, Mayba O, Yaylaoglu M, Gnad F, Firestein R. CCAT1 is an enhancer-templated RNA that predicts BET sensitivity in colorectal cancer. J Clin Invest. 2016;126:639-52.

15. Zhang MX, Zhang J, Zhang H, Tang H. miR-24-3p Suppresses Malignant Behavior of Lacrimal Adenoid Cystic Carcinoma by Targeting PRKCH to Regulate p53/p21 Pathway. PLoS One. 2016;11:e0158433.

16. Wang S, Pan Y, Zhang R, Xu T, Wu W, Zhang R, Wang C, Huang H, Calin CA, Yang H, Claret FX. HsamiR-24-3p increases nasopharyngeal carcinoma radiosensitivity by targeting both the 3'UTR and 5'UTR of Jab1/CSN5. Oncogene. 2016;35:6096-108.

17. Li X, Han X, Wei P, Yang J, Sun J. Knockdown of IncRNA CCAT1 enhances sensitivity of paclitaxel in prostate cancer via regulating miR-24-3p and FSCN1. Cancer Biol Ther. 2020;21:452-62.

18. Kureishy N, Sapountzi V, Prag S, Anilkumar N, Adams JC. Fascins, and their roles in cell structure and function. Bioessays. 2002;24:350-61.

19. Adams JC. Roles of fascin in cell adhesion and motility. Curr Opin Cell Biol. 2004;16:590-6.

20. Hashimoto Y, Ito T, Inoue H, Okumura T, Tanaka E, Tsunoda S, Higashiyama M, Watanabe G, Imamura M, Shimada Y. Prognostic significance of fascin overexpression in human esophageal squamous cell carcinoma. Clin Cancer Res. 2005;11:2597-605.

21. Li Y, Jing F, Ding Y, He Q, Zhong Y, Fan C. Long noncoding RNA CCAT1 polymorphisms are associated with the risk of colorectal cancer. Cancer Genet. 2018;222-223:13-9.

22. Zhang $\mathrm{Y}, \mathrm{Hu} \mathrm{H}$. Long non-coding RNA CCAT1/miR-218/ZFX axis modulates the progression of laryngeal squamous cell cancer. Tumour Biol. 2017;39:1010428317699417.

23. Alaiyan B, llyayev N, Stojadinovic A, Izadjoo M, Roistacher M, Pavlov V, Tzivin V, Halle D, Pan H, Trink B, Gure AO, Nissan A. Differential expression of colon cancer associated transcript1 (CCAT1) along the colonic adenoma-carcinoma sequence. BMC Cancer. 2013;13:196.

24. Takeda M, Mizokami A, Mamiya K, Li YQ, Zhang J, Keller ET, Namiki M. The establishment of two paclitaxel-resistant prostate cancer cell lines and the mechanisms of paclitaxel resistance with two cell lines. Prostate. 2007;67:955-67. 
25. Karnak D, Xu L. Chemosensitization of prostate cancer by modulating Bcl-2 family proteins. Curr Drug Targets. 2010;11:699-707.

26. Mishra PJ, Song B, Mishra PJ, Wang Y, Humeniuk R, Banerjee D, Merlino G, Ju J, Bertino JR. MiR-24 tumor suppressor activity is regulated independent of p53 and through a target site polymorphism. PLoS One. 2009;4:e8445.

27. Fang Z, Tang J, Bai Y, Lin H, You H, Jin H, Lin L, You P, Li J, Dai Z, Liang X, Su Y, Hu Q, Wang F, Zhang ZY. Plasma levels of microRNA-24, microRNA-320a, and microRNA-423-5p are potential biomarkers for colorectal carcinoma. J Exp Clin Cancer Res. 2015;34:86.

28. Adams JC. Characterization of cell-matrix adhesion requirements for the formation of fascin microspikes. Mol Biol Cell. 1997;8:2345-63.

29. Chen L, Yang S, Jakoncic J, Zhang JJ, Huang XY. Migrastatin analogues target fascin to block tumour metastasis. Nature. 2010;464:1062-6.

30. Huang FK, Han S, Xing B, Huang J, Liu B, Bordeleau F, Reinhart-King CA, Zhang JJ, Huang XY. Targeted inhibition of fascin function blocks tumour invasion and metastatic colonization. Nat Commun. 2015;6:7465.

31. Hashimoto Y, Parsons M, Adams JC. Dual actin-bundling and protein kinase C-binding activities of fascin regulate carcinoma cell migration downstream of Rac and contribute to metastasis. Mol Biol Cell. 2007;18:4591-602.

32. Ma Y, Faller WJ, Sansom OJ, Brown ER, Doig TN, Melton DW, Machesky LM. Fascin expression is increased in metastatic lesions but does not correlate with progression nor outcome in melanoma. Melanoma Res. 2015;25:169-72.

\section{Tables}

Table 1 was omitted by the authors in this version of the paper.

\section{Figures}




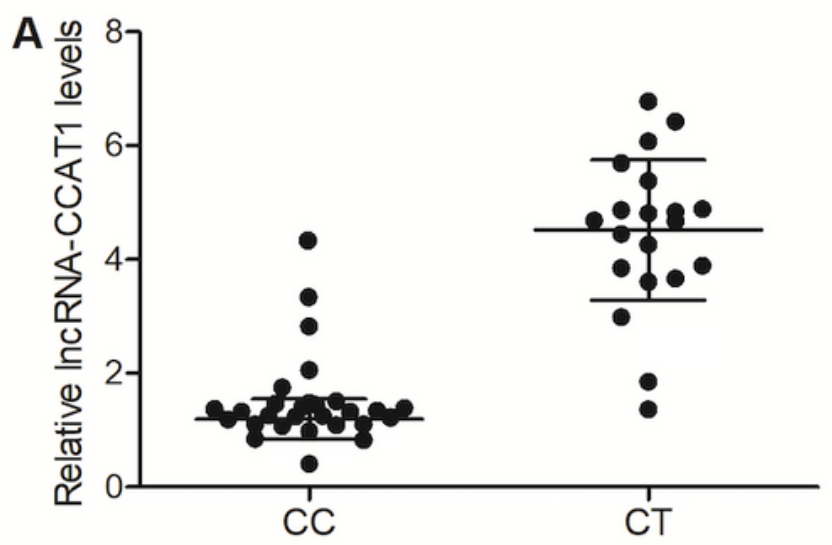

B

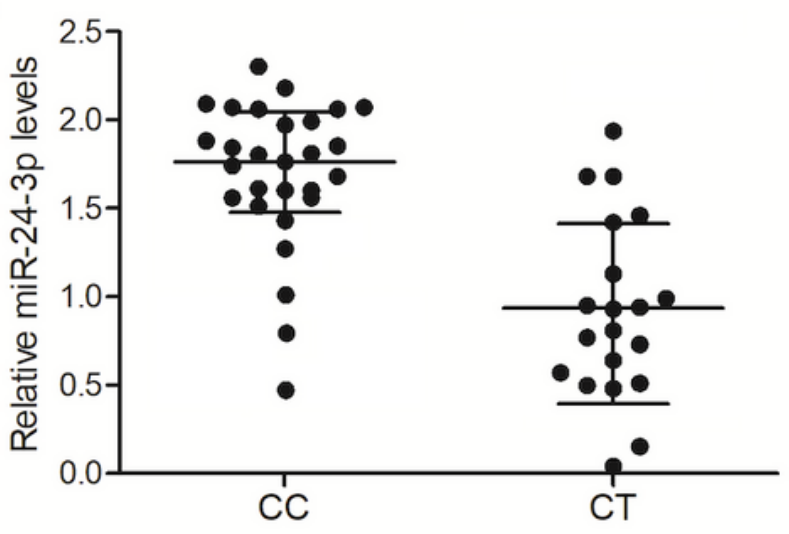

C

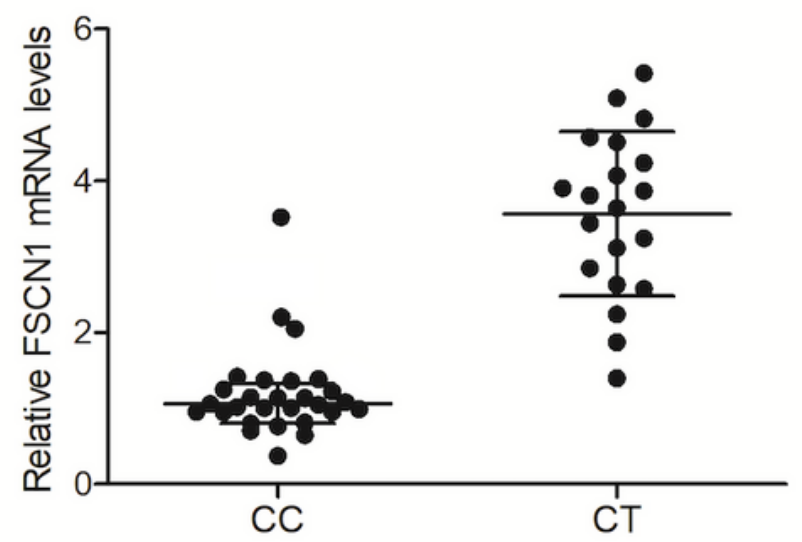

Figure 1

Expression of IncRNA-CCAT1, miR-24-3p and FSCN1 mRNA in colon cancer patients with different genotypes A: The expression of IncRNA-CCAT1 in the CC group and the CT group. B: The expression of miR-24-3p in the CC group and the CT group. C: The expression of FSCN1 mRNA in the CC group and the CT group. 

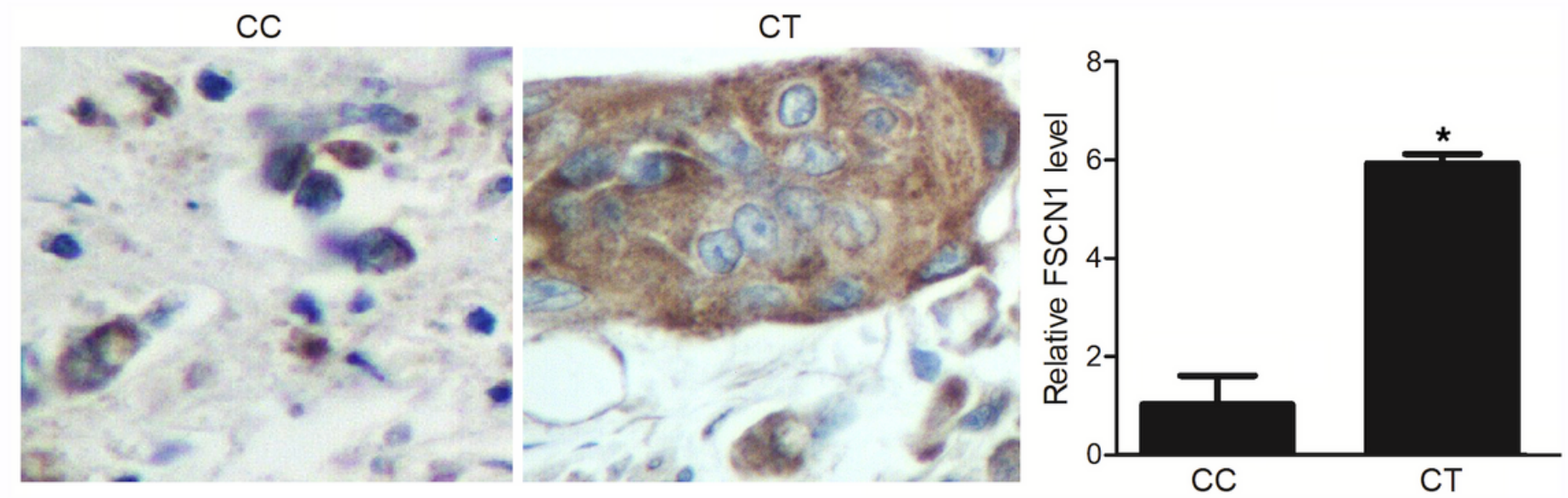

Figure 2

IHC assay indicated the expression of FSCN1 protein in colon cancer patients with CC and CT genotypes (* $p$-value $<0.05$ compared with CC group).

A
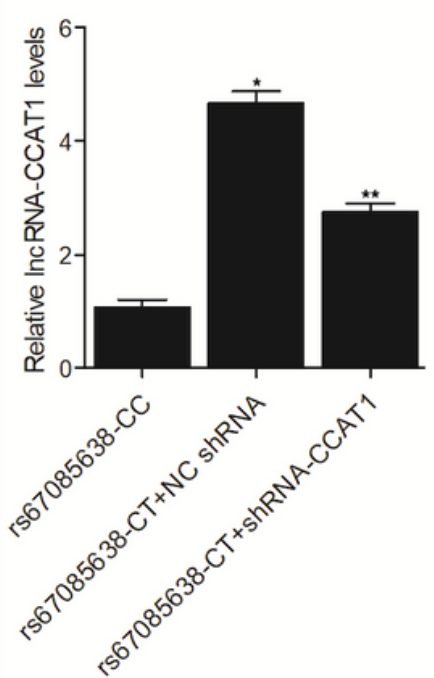

B
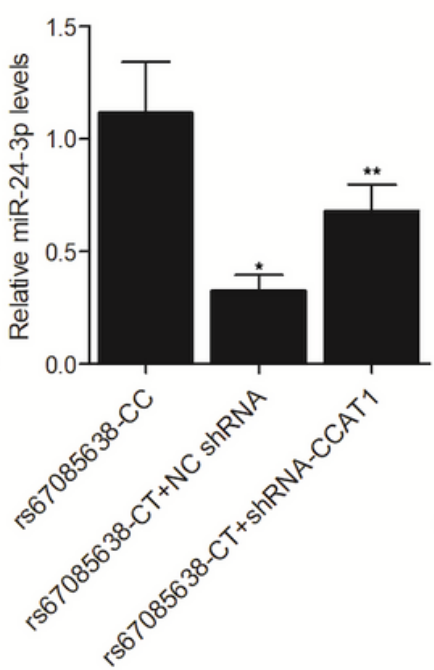

C
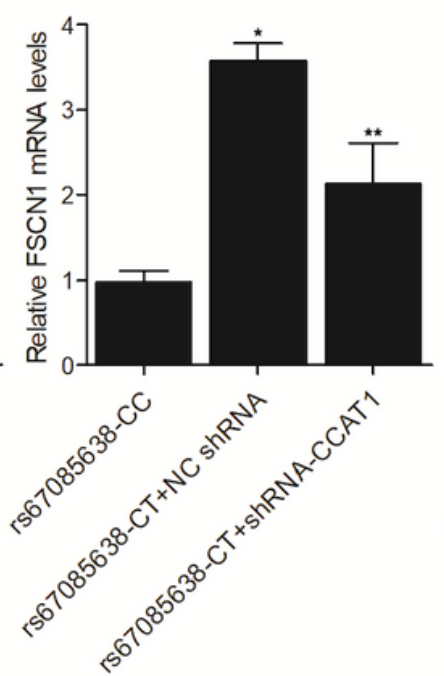
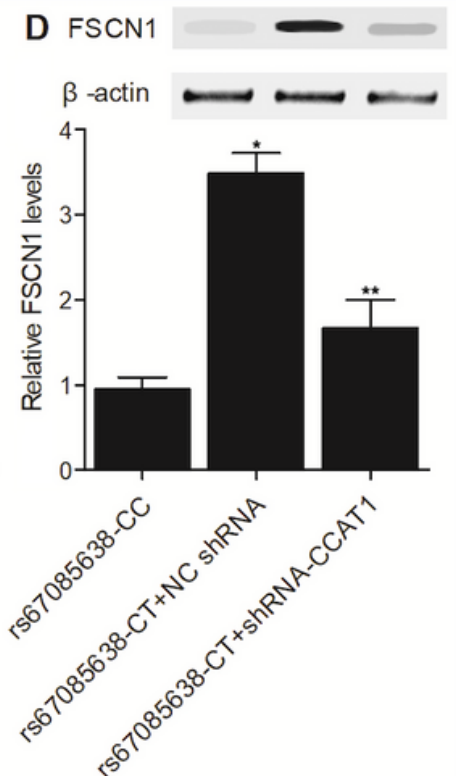

\section{Figure 3}

Expression of IncRNA-CCAT1, miR-24-3p and FSCN1 mRNA in colon cancer cells collected from patients with different genotypes (* $p$-value $<0.05$ compared with rs67085638-CC group; ** $p$-value $<0.05$ compared with rs67085638-CT+NC shRNA group). A: The expression of IncRNA-CCAT1 in the rs67085638-CC group, the rs67085638-CT+NC shRNA group and the rs67085638-CT+shRNA-CCAT1 group. B: The expression of miR-24-3p in the rs67085638-CC group, the rs67085638-CT+NC shRNA group and the rs67085638-CT+shRNA-CCAT1 group. C: The expression of FSCN1 mRNA in the rs67085638-CC group, the rs67085638-CT+NC shRNA group and the rs67085638-CT+shRNA-CCAT1 group. D: The 
expression of FSCN1 protein in the rs67085638-CC group, the rs67085638-CT+NC shRNA group and the rs67085638-CT+shRNA-CCAT1 group.
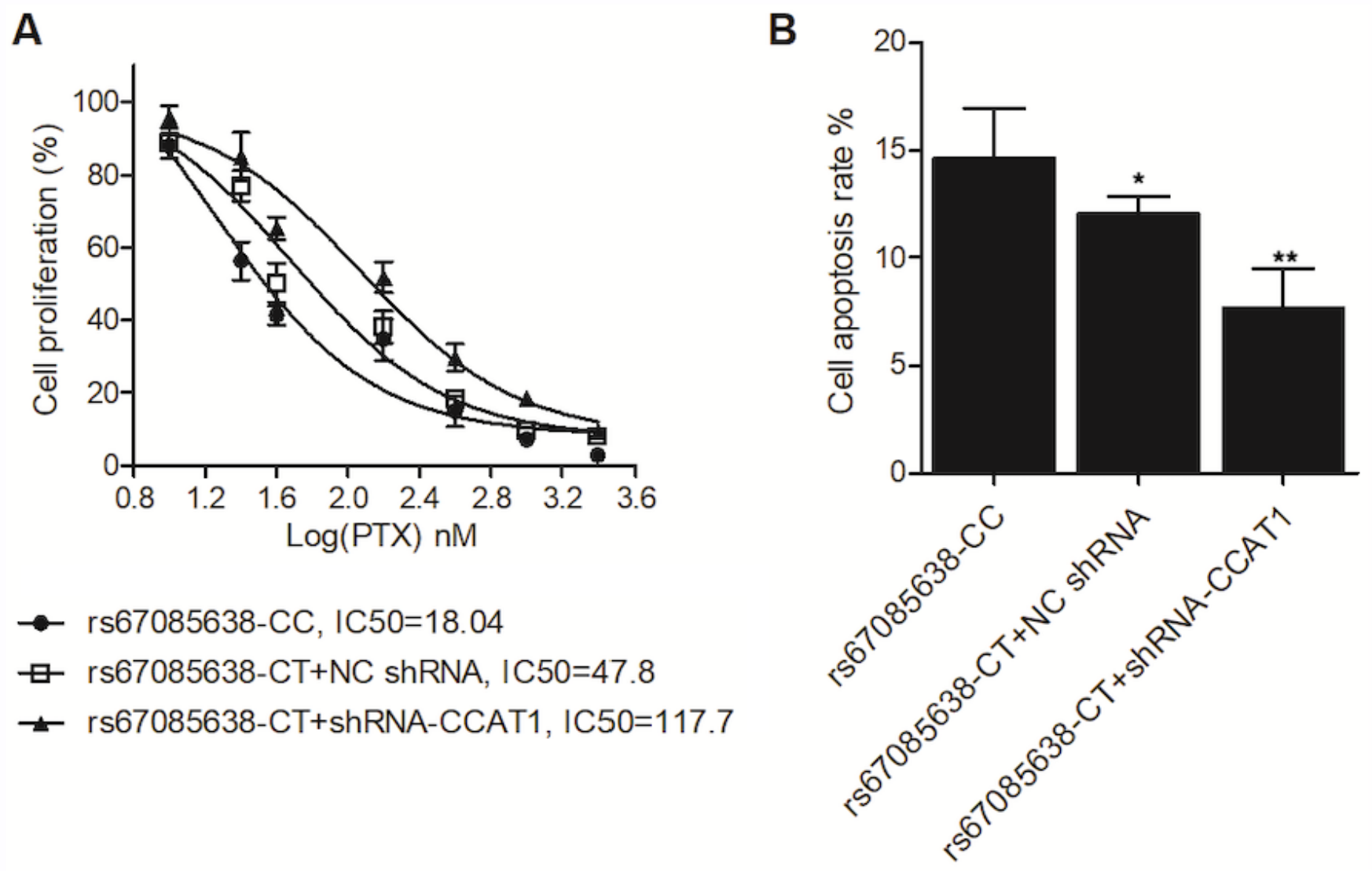

\section{Figure 4}

Survival and apoptosis induced by PTX treatment of colon cancer cells collected from patients with different genotypes ( ${ }^{*} p$-value $<0.05$ compared with rs67085638-CC group; ** $p$-value $<0.05$ compared with rs67085638-CT+NC shRNA group). A: The survival rate of the rs67085638-CC group, the rs67085638-CT+NC shRNA group and the rs67085638-CT+shRNA-CCAT1 group treated with PTX at different concentrations. B: The apoptosis rate of the rs67085638-CC group, the rs67085638-CT+NC shRNA group and the rs67085638-CT+shRNA-CCAT1 group treated with PTX at different concentrations. 
A

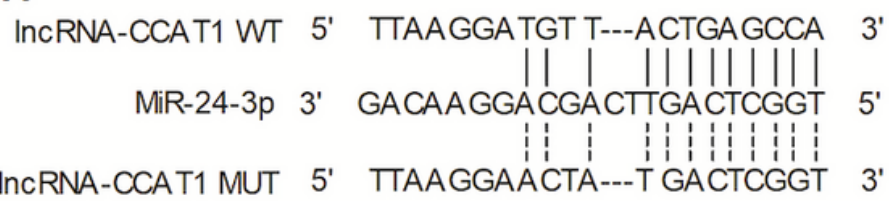

B

FSCN1 WT 5' AGAAAAUGACCAAAU 3'

MiR-24-3p 3' ACUUCCCCUGGUUU 5'

II 11111

FSCN1 MUT 5' AGAAAAUGAAACAAU 3'
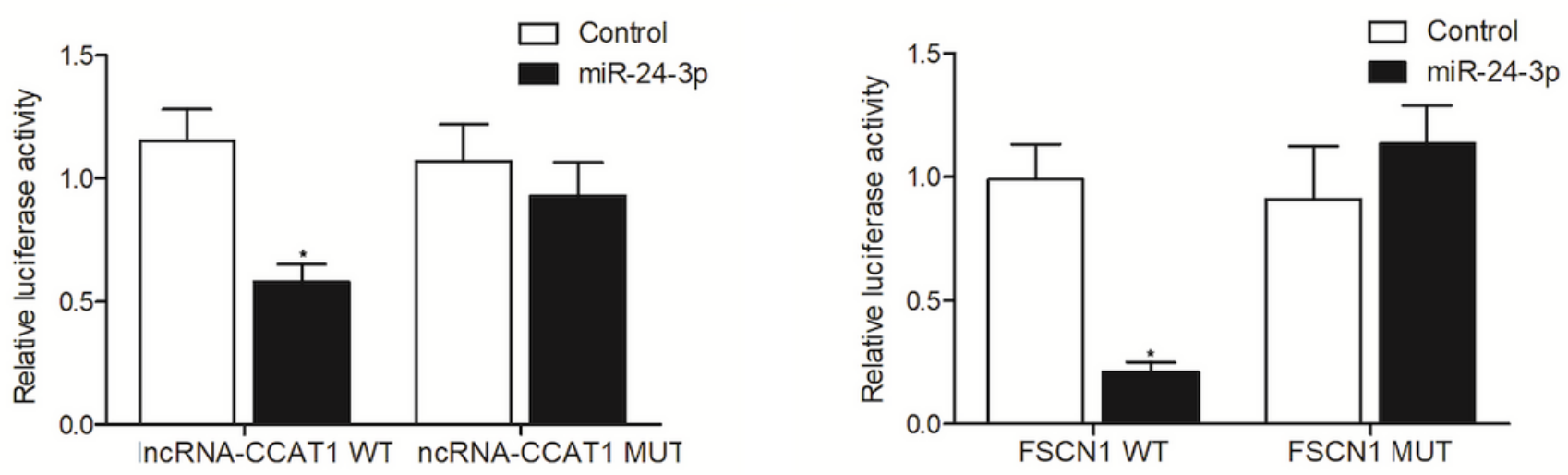

C

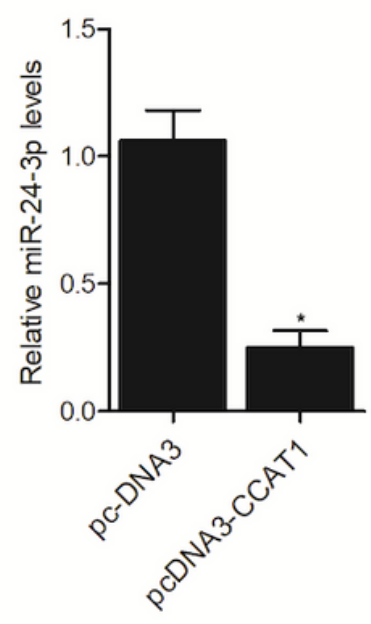

D

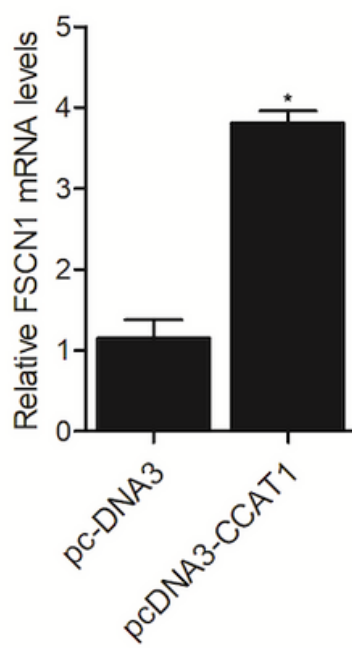

E

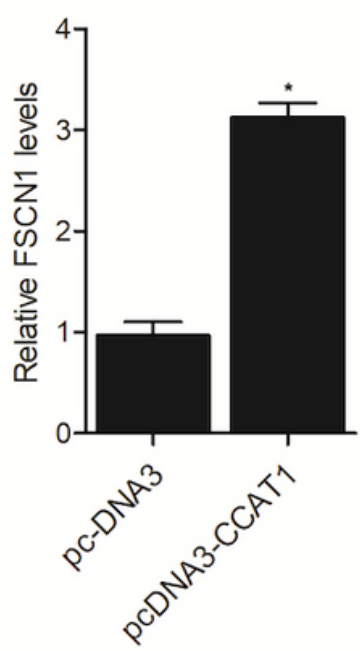

FSCN1

$\beta$-actin

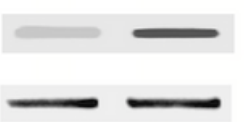

\section{Figure 5}

The CCAT1/miR-24-3p/FSCN1 signaling pathway was established in CACO-2 cells A: Sequence analysis and luciferase assay of miR-24-3p and IncRNA-CCAT1 (* $p$-value $<0.05$ compared with IncRNA-CCAT1 WT + control group). B: Sequence analysis and luciferase assay of miR-24-3p and FSCN1 3'UTR (* $p$ value $<0.05$ compared with FSCN1 WT + control group). C: Expression of miR-24-3p in the pcDNA3 group and the pcDNA3-CCAT1 group ( ${ }^{*}$-value $<0.05$ compared with pcDNA3 group). D: Expression of FSCN1 mRNA in the pcDNA3 group and the pcDNA3-CCAT1 group ( ${ }^{*}$-value $<0.05$ compared with pcDNA3 group). E: Expression of FSCN1 protein in the pcDNA3 group and the pcDNA3-CCAT1 group (* $p$-value < 0.05 compared with pcDNA3 group). 

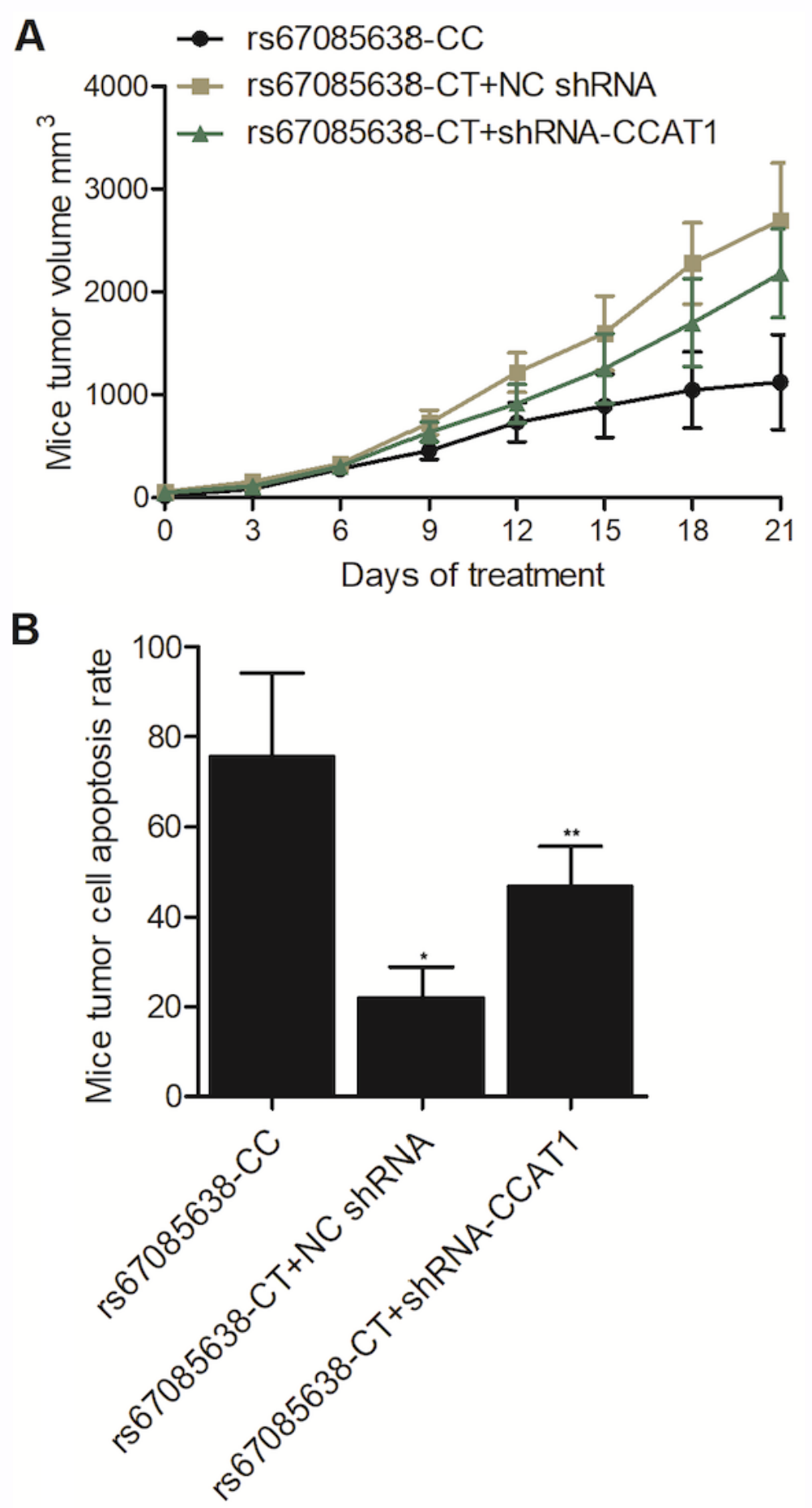

\section{Figure 6}

The tumor volume and the tumor cell apoptosis rate of the xenograft in the rs67085638-CC mice, the rs67085638-CT+NC shRNA mice and the rs67085638-CT+shRNA-CCAT1 mice ( ${ }^{*}$-value $<0.05$ compared with rs67085638-CC group; ** $p$-value $<0.05$ compared with rs67085638-CT+NC shRNA group). A: The tumor volume in the rs67085638-CC mice, the rs67085638-CT+NC shRNA mice and the rs67085638- 
CT+shRNA-CCAT1 mice. B: The tumor cell apoptosis rate in the rs67085638-CC mice, the rs67085638$\mathrm{CT}+\mathrm{NC}$ shRNA mice and the rs67085638-CT+shRNA-CCAT1 mice.

A

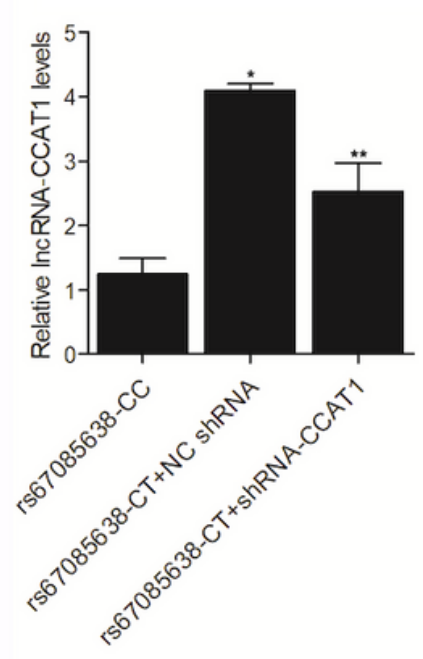

B
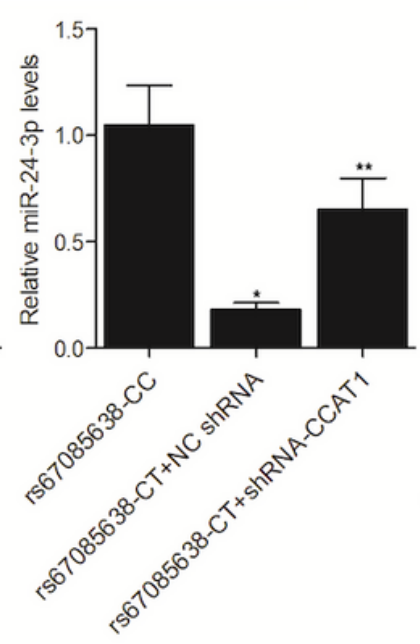

C
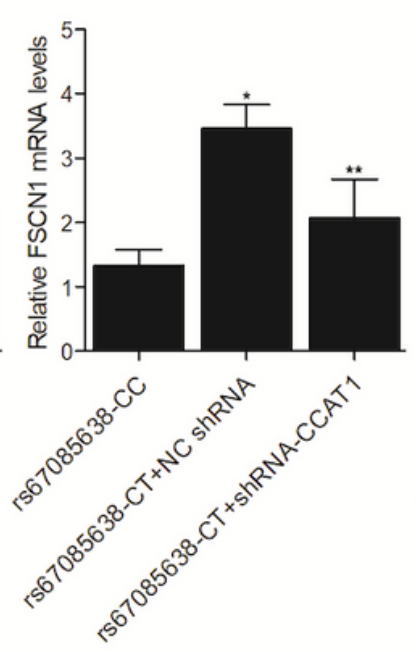
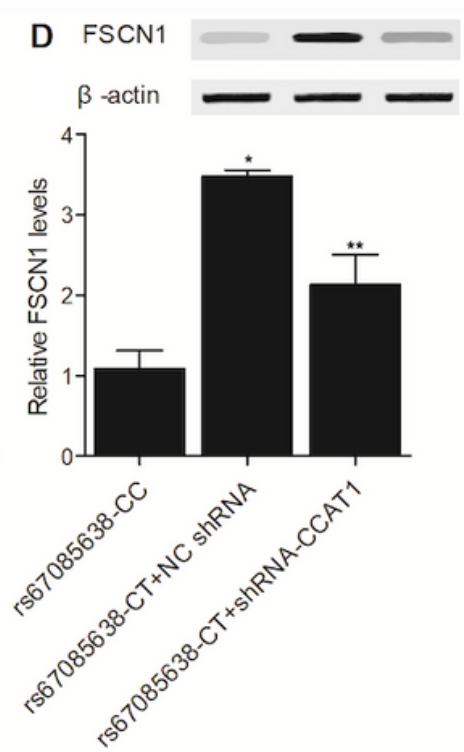

\section{Figure 7}

Expression of IncRNA-CCAT1, miR-24-3p and FSCN1 mRNA in xenograft mice (* $p$-value $<0.05$ compared with rs67085638-CC group; ** $p$-value $<0.05$ compared with rs67085638-CT+NC shRNA group). A: The expression of IncRNA-CCAT1 in the rs67085638-CC mice, the rs67085638-CT+NC shRNA mice and the rs67085638-CT+shRNA-CCAT1 mice. B: The expression of miR-24-3p in the rs67085638-CC mice, the rs67085638-CT+NC shRNA mice and the rs67085638-CT+shRNA-CCAT1 mice. C: The expression of FSCN1 mRNA in the rs67085638-CC mice, the rs67085638-CT+NC shRNA mice and the rs67085638CT+shRNA-CCAT1 mice. D: The expression of FSCN1 protein in the rs67085638-CC mice, the rs67085638$\mathrm{CT}+\mathrm{NC}$ shRNA mice and the rs67085638-CT+shRNA-CCAT1 mice. 